\title{
Outflow changes in normal eyes after closed-angle glaucoma
}

\author{
R. MAPSTONE \\ From St. Paul's Eye Hospital, Liverpool
}

SUMMARY Twenty-four patients with spontaneous acute closed-angle glaucoma in one eye were selected for study. All 24 eyes had a peripheral iridectomy, were normotensive, and had no gonioscopically visible peripheral anterior synechiae. Of the 24 contralateral eyes 14 gave a positive response to provocative tests and had peripheral iridectomy. The remaining 10 eyes did not give positive responses to the tests and were on no treatment.

The 24 pairs of eyes were provoked with pilocarpine and phenylephrine. Tonography was performed at the start of the test, $1 \frac{1}{2}$ hours later, and at its termination. At the start of the test the 24 eyes that had had spontaneous closed-angle glaucoma showed a higher pressure and lower outflow facility than the 24 contralateral eyes. This difference disappeared as the test progressed. It was concluded that apparently normal eyes-after an acute attack-do none the less show a significant degree of damage to the outflow system. Ten pairs of eyes from 10 normal persons were provoked in a similar fashion and at no point did a significant difference appear between right and left eyes.

After an attack of spontaneous acute closed-angle glaucoma and subsequent iridectomy some eyes still need treatment. The cause of this residual increase in pressure is either permanent occlusion of part or all of the angle by peripheral anterior synechiae, or (it is postulated), if none are visible, damage to the trabecular meshwork.

If an acute rise in pressure can cause so much damage to the outflow system-in the absence of anterior synechiae - that pressure remains high, then it would seem reasonable to suppose that it could also produce an outflow deficit insufficient to require treatment. Therefore some eyes that are normotensive after iridectomy would have a reduced outflow facility. An investigation of that hypothesis is reported here.

\section{Material and methods}

Twenty-four patients were selected on the following criteria: (a) An attack of acute closed-angle glaucoma in one eye treated by a peripheral iridectomy. Subsequently, all eyes were normotensive on no treatment and had had no gonioscopically visible peripheral anterior synechiae. (b) The 24 contralateral eyes had no evidence of closed-angle glaucoma and

Address for reprints: Mr R. Mapstone, St. Paul's Eye Hospital, Old Hall Street, Liverpool L3 9PF were provoked with simultaneous pilocarpine and phenylephrine (Mapstone, 1976). If the test was positive a peripheral iridectomy was done (14 eyes), if negative no treatment was given (10 eyes).

The 24 patients were then provoked as follows: At zero hours intraocular pressures were recorded and tonography done on the right eye. Immediately afterwards tonography was done on the left eye. Pilocarpine $2 \%$ and phenylephrine $10 \%$ drops were then instilled into both eyes. Approximately 45 minutes later pressure was recorded and phenylephrine drops $10 \%$ were instilled. After the lapse of another 45 minutes pressures were recorded, tonography of right and left eyes was done, and pilocarpine $2 \%$ and phenylephrine $10 \%$ drops were instilled. One hour later, that is approximately $2 \frac{1}{2}$ hours after the start of the test, pressures were measured and tonography was repeated.

Ten patients with no evidence of glaucoma were provoked as described above.

The statistical tests used were paired $t$ tests.

\section{Results}

PROVOCATIVE TESTS ON 24 PATIENTS

Fig. $1 a$ records the result of provoking 24 eyes which had had an acute attack of closed-angle glaucoma. The first dose of pilocarpine and phenylephrine produced a significant fall in pressure, from a mean of 

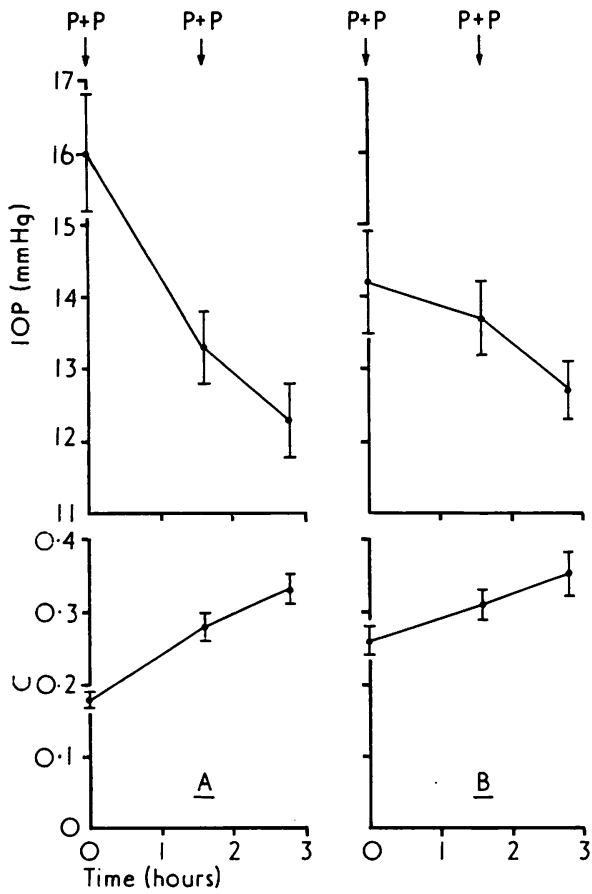

Fig. 1a Response of 24 eyes that had had a spontaneous attack of closed-angle glaucoma and subsequent peripheral iridectomy to provocative testing with simultaneous pilocarpine and phenylephrine. Mean and standard error recorded. $P$ and $P=$ pilocarpine and phenylephrine

Fig. $1 b$ Response of 24 contralateral eyes that had not had a spontaneous attack of closed-angle glaucoma to piovocative testing with simultaneous pilocarpine and phenylephrine. Mean and standard error recorded

$16.0 \mathrm{mmHg}$ to a mean of $13.3 \mathrm{mmHg}(\mathrm{P}<0.001)$; and a significant increase in $\mathrm{C}$, from a mean of $0 \cdot 18$ to a mean of $0.28(\mathrm{P}<0.001)$. The second dose of pilocarpine and phenylephrine also produced a significant decrease in pressure $(\mathrm{P}<0.01)$ and a significant increase in $\mathrm{C}(\mathrm{P}<0.05)$.

Fig. $1 b$ records the results of provoking the 24 contralateral eyes that had not had a spontaneous attack of acute closed-angle glaucoma. The first dose of pilocarpine and phenylephrine produced an insignificant decrease in pressure from a mean of 14.2 to a mean of $13.7 \mathrm{mmHg}$, but a significant increase in $C$, from a mean of 0.26 to a mean of $0.31(P<0.01)$. The second dose of pilocarpine and phenylephrine produced a significant decrease in pressure $(\mathrm{P}<0.05)$ and a significant increase in $\mathrm{C}(\mathrm{P}<0.01)$.

$\cdots$ A comparison of Figs. $1 a$ and $1 b$ shows that before the test the eyes that had had a spontaneous acute attack had a significantly higher pressure $(P<0.001)$ and a significantly lower $C(P<0.001)$ than the contralateral normal eyes. At the time of instillation of the second dose of pilocarpine and phenylephrine there was no significant difference in pressure between the two groups of eyes, but $\mathrm{C}$ in the eyes with an acute attack was still significantly lower $(P<0.05)$. At the termination of the provocative test there was no significant difference between the two groups of eyes, in either pressure or outflow facility.

PROVOCATIVE TESTS ON 10 NORMAL PATIENTS Fig. 2 records the result of provoking 10 normal patients with pilocarpine and phenylephrine. Both the decrease in pressure and increase in $\mathrm{C}$ in both eyes is significant $(P<0.001)$, but no significant difference appeared between right and left eyes at any stage during the test.

\section{Discussion}

The interpretation of provocative test results in eyes that have had a peripheral iridectomy requires care. Although at the start of the test the angles were open and free of synechiae, the first dose of pilocarpine and phenylephrine moves the pupil to mid-dilatation and might produce partial angle closure with a decrease in $\mathrm{C}$ and increase in pressure. There are reports that this can happen. For example, Lowe (1964) dilated pupils (that had previously had a peripheral iridectomy for closed-angle glaucoma) with parasympatholytic drugs and in $19 \%$ produced a

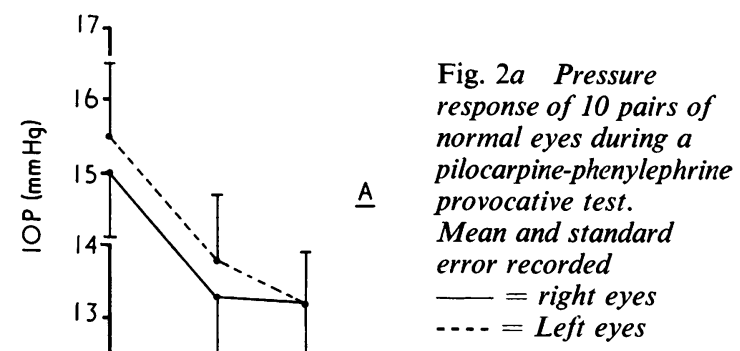

Fig. $2 b$ Outflow response of 10 pairs of normal eyes during a pilocarpine-phenylephrine provocative test.

Tonography done on right eye and immediately afterwards on the left eye. Mean and standard error recorded 
significant increase in pressure. In a later rejoinder (Lowe, 1969) to a paper by Harris and Galin (1969) he states, 'high pressure rises occurred only when angles were totally occluded by iris folding.' In contrast Harris and Galin (1969) performed a similar investigation and produced a significant pressure rise in $21 \%$. But the pressure increase occurred only in patients who had previously had a raised tension (Lowe's were all normotensive), and 'no angle was felt to be occluded at any time in this study'. They concluded that a decrease in ciliary muscle tone induced a reduction in outflow facility.

It has been shown (Mapstone, 1977) that the response of normal eyes to the simultaneous instillation of pilocarpine and phenylephrine is to develop a significant increase in outflow. Furthermore (Mapstone, 1977) 60\% of contralateral eyes at risk to closed-angle glaucoma (which did not develop a positive provocative test) showed a significant reduction in outflow at some stage during the test which was probably due to partial angle closure. Of the 24 eyes described here that had a spontaneous acute attack 2 showed a reduction in outflow at some stage during the provocative test. In the 24 contralateral eyes 6 produced a similar result, 4 of which had not had peripheral iridectomies.

The 24 eyes with spontaneous closed-angle glaucoma had at the start of the test a significantly higher pressure and lower outflow than the 24 contralateral eyes. But the effect of pilocarpine and phenylephrine as the test progressed was to cancel this difference, so that at the end both groups of eyes had equivalent pressures and outflow.

The first question to be asked is what is the cause of the asymmetry, and there would seem to be five main possibilities. Firstly, it could be argued that the presence of previous open-angle glaucoma is the cause of the differences. This is almost certainly not so, since it would be necessary to postulate its presence in all 24 eyes, and unilaterally too. Secondly, the effect of a raised pressure on outflow resistance could be advanced in explanation. Ellingsen and Grant $(1971 \mathrm{a}, \mathrm{b})$ have shown that outflow resistance is to some extent pressure-dependent-that is, the higher the pressure the lower is outflow-and Johnstone and Grant (1973) and Grierson and Lee (1974) have postulated a mechanism. In the 24 pairs of eyes, however, the pressure difference is small. It is therefore unlikely that this mechanism was of much significance. Thirdly, numerous authors have indicated a 'consensual' effect when tonography is done on both eyes consecutively. There seems to be no agreement as to the direction of the effect (i.e., whether an increase or decrease in $\mathrm{C}$ is produced in the contralateral eye). Provocative tests done on 10 normal patients indicate no significant difference between right and left eyes. This mechanism therefore would appear not to be contributory. Fourthly, it is possible that partial-angle closure might explain some of the observed discrepancy. Again this is unlikely, since the angles were open at the start of the test, and, as mentioned above, reductions in $\mathrm{C}$ were not very common.

The final possibility is that an acute rise in intraocular pressure produced damage to the outflow pathways which significantly reduced $\mathrm{C}$ and increased pressure relative to the contralateral eye. These changes were reversed by the provocative test.

The nature of the damage to the outflow system is of interest. Grant and Trotter (1955) and Becker and Constant (1956) have shown that outflow values are similar in eyes in vivo and when perfused post mortem. Metabolic inhibitors and wide variations in temperature (Van Buskirk and Grant, 1974) also have little influence on outflow in excised eyes. Finally, Van Buskirk and Grant (1973) showed that mechanical traction (lens depression) on angle structures increases outflow. If, therefore, outflow changes are determined largely by mechanical factors, it would seem that an acute rise in pressure induces a mechanical change that is completely reversible by a mechanical process (Bárány, 1966).

Whatever may be the explanation, it is apparent that eyes judged to be clinically normal after an attack of closed-angle glaucoma do none the less show a significant reduction in outflow and increase in pressure relative to the contralateral eye. To say that this is a secondary change-which it is-but therefore because of this not comparable to the change in open-angle glaucoma, begs the question, since it assumes that the reduction in outflow in open-angle glaucoma is in some way primary (although it must have a cause). Open-angle glaucoma is 'primary' only because the causal mechanism(s) is unknown. Again, there is no reason to suppose that there must be one unique 'cause' for reduced $\mathrm{C}$ values in open-angle glaucoma, and these results raise the possibility that one such cause may be an increase in pressure produced by an angle-closing mechanism.

\section{I thank Mrs E. Tubb for secretarial help.}

\section{References}

Bárány, E. H. (1966). The mode of action of miotics on outflow resistance: a study of pilocarpine in the vervet monkey Cercopithecus ethiops. Transactions of the Ophthalmological Societies of the United Kingdom, 86, 539-578.

Becker, B., and Constant, M. A. (1956). The facility of aqueous outflow. Archives of Ophthalmology, 55, 305-312. Ellingsen, B. A., and Grant, W. M. (1971a). The relationship 
of pressure and aqueous outflow in enucleated human eyes. Investigative Ophthalmology, 10, 430-437.

Ellingsen, B. A., and Grant, W. M. (1971b). Influence of intraocular pressure and trabeculotomy on aqueous outflow in enucleated monkey eyes. Investigative Ophthalmology, 10, 705-709.

Grant, W. M., and Trotter, R. R. (1955). Tonographic measurements in enucleated eyes. Archives of Ophthalmology, 53, 191-200.

Grierson, I., and Lee, W. R. (1974). Changes in the monkey outflow apparatus at graded levels of intraocular pressure. Experimental Eye Research, 19, 21-33.

Harris, L. S., and Galin, M. A. (1969). Cycloplegic provocative testing. Archives of Ophthalmology, 81, 356-358.

Johnstone, M. A., and Grant (1973). Pressure dependent changes in structures of the aqueous outflow system in human and monkey eyes. American Journal of Ophthalmology, 75, 365-383.
Lowe, R. F. (1964). Primary angle closure glaucoma. American Journal of Ophthalmology, 57, 931-938.

Lowe, R. F. (1969). Cycloplegic provocative testing. Archives of Ophthalmology, 82, 294.

Mapstone, R. (1976). Provocative tests in closed-angle glaucoma. British Journal of Ophthalmology, 60, 115-120.

Mapstone, R. (1977). Normal response to pilocarpine and phenylephrine. British Journal of Ophthalmology. 61, 510511.

Mapstone, R. (1977). Partial angle closure. British Journal of Ophthalmology, 61, 525-530.

Van Buskirk, E. M., and Grant, W. M. (1973). Lens depression and aqueous outflow in enucleated primate eyes. American Journal of Ophthalmology, 76, 632-640.

Van Buskirk, E. M., and Grant, W. M. (1974). Influence of temperature and the question of involvement of cellular metabolism in aqueous outflow. American Journal of Ophthalmology, 77, 565-572. 